\title{
Polydiphenylamine/Zeolite Y Composites and Electrical Conductivity Responses toward Halogenated Hydrocarbons
}

\author{
Tharaporn Permpool ${ }^{\mathrm{a}}$, Anuvat Sirivat ${ }^{\mathrm{a} *}$, Darunee Aussawasathien ${ }^{\mathrm{b}}$, Ladawan Wannatong $^{\mathrm{c}}$ \\ aThe Petroleum and Petrochemical College, Chulalongkorn University, Bangkok, 10330, Thailand \\ ${ }^{\mathrm{b}}$ National Metal and Materials Technology center, National Science and Technology Development Agency, \\ Ministry of Science and Technology, Pathumthani, 12120, Thailand \\ ${ }^{\mathrm{c}}$ Faculty of Engineering, King Mongkut's University of Technology North Bangkok, \\ Bangkok, 10800, Thailand
}

Received: November 8, 2012; Revised: February 8, 2013

\begin{abstract}
Composites of polydiphenylamine (D-PDPA) and zeolite $\mathrm{Y}$ with $\mathrm{H}^{+}$as the cation $\left(\mathrm{Y} \_\mathrm{H}^{+}\right)$have been fabricated to be used as a sensing material towards non-halogenated and halogenated solvents (hexane, dichloromethane, 1, 2-dichloroethane, chloroform). These composites are toxic towards human and environment and are widely used as solvents in various industries. Polydiphenylamine, zeolite $\mathrm{Y}$, and their composites are characterized by Fourier transform infrared spectroscopy, scanning electron microscopy, particle size analysis, surface area, and pore size analysis. The effects of the $\mathrm{Si} / \mathrm{Al}$ ratio, zeolite content, and vapor concentrations are investigated. The electrical conductivity sensitivity of the composites towards the solvents is higher than the pristine D-PDPA by $\sim 1$ order of magnitude. The composites can discriminate a non-halogenated solvent from halogenated solvents. They possess maximum electrical conductivity sensitivity values towards dichloromethane, but the composites do not respond to hexane. Generally, the sensitivity of the composites increases with increasing zeolite content and vapor concentration. The interactions between the composites and the vapors are investigated by FT-IR spectroscopy and UV-Vis spectroscopy. A mechanism for the interaction between the composites and the solvents is proposed.
\end{abstract}

Keywords: Conductive polymer, electrical conductivity, polydiphenylamine, Zeolite Y, halogenated hydrocarbon

\section{Introduction}

Volatile organic compounds (VOCs) are organic compounds that easily evaporate at normal pressure and room temperature. The VOCs are widely used in daily products and used as solvents in various industries ${ }^{1}$. Nevertheless, the toxicity of these chemical vapors causes serious environmental and human health concerns. The VOCs are divided into two groups; non-halogenated hydrocarbons, and halogenated hydrocarbons. Nonhalogenated hydrocarbons are volatile hydrocarbons that do not contain a chlorine atom within the molecule. Normally, this group is found in daily life products such as plastics, cleaning solvents, and paints, and it can affect human health through the respiratory system. Examples of non-halogenated hydrocarbons are aliphatic hydrocarbons, aromatic hydrocarbons, alcohol, aldehyde, and ketones. The other type of VOCs is halogenated hydrocarbons which consist of a chlorine atom within the molecule. They are used in dry cleaning, metal cleaning, furniture making, thermoplastics production, degreasing, printing, paper and textile production, and paint removal ${ }^{1}$. They are clear liquids, and vaporize at room temperature $e^{2-4}$. Examples of halogenated solvents are trichloroethylene, perchloroethylene, methylene chloride, ethylene chloride,

*e-mail: anuvat.s@chula.ac.th carbon tetrachloride, chloroform, and methyl chloroform. The toxicity of these chemical vapors is more severe to human health than non-halogenated hydrocarbons. The solvents pose a major source of environmental problems such as stratospheric ozone depletion, smog formation, acid rain production, and global warming. Health problems caused by exposing humans to these solvents are systemic, immunological, neurological, reproductive, developmental, and genotoxic and carcinogenic in nature; which can lead to death ${ }^{5}$. The severity of the chemicals on human health depends on the concentration and exposure levels. The National Institute of Occupational Safety and Health (NIOSH) defined the limit for personal exposure to the chemical that cause to death or immediate or delayed permanent adverse health effects as the immediately dangerous to life or health concentrations (IDLHs) ${ }^{6}$. For instance, the exposure level of chloroform that leads to death in humans is $\sim 40,000 \mathrm{ppm}^{7}$. The level of chloroform that leads to human loss of responsiveness, loss of skeleton muscle reflex, and decreased stress response is less than $22,500 \mathrm{ppm}^{5,8}$. The deadly inhalation concentration for methylene chloride, or dichloromethane (DCM), in a study of animals, was $\sim 3,000 \mathrm{ppm}^{9}$. Hence, effective sensory systems are required to identify the presence of these 
solvents. One development of sensing materials has focused on conductive polymers due to their unique properties. They are: light weight, easy to synthesize, possess high sensitivity and short response time, and have good mechanical properties over metal oxides ${ }^{10-12}$. Polydiphenylamine (PDPA) is an N-aryl substituted derivative of polyaniline and it shows better mechanical strength, electrochemical, conductivity, and electro chromic properties than polyaniline ${ }^{13-16}$. Additionally, PDPA has been used as a sensing material for many sensory systems: $\mathrm{pH}$ sensors, $\mathrm{CO}$ sensors, and glucose biosensors ${ }^{17-19}$. Another group of materials that can also be used as sensing materials are zeolites. Zeolites are aluminosilicate minerals that have micro porous cages within their crystal structures. Due to their unique structures, zeolites can act as molecular sieves to separate different types of molecules. Thus, zeolites can be used as a catalyst support, an absorbent for moisture and toxic gases, and for ion exchange. Recently, there have been several studies on conductive polymer/zeolite composites as gas or vapor sensing materials ${ }^{20-22}$.

This work is aimed at fabricating the composites between D-PDPA and zeolite $\mathrm{Y}$ with $\mathrm{H}^{+}$as a cation $\left(\mathrm{Y} \_\mathrm{H}^{+}\right)$to discriminate between the non-halogenated and halogenated hydrocarbon solvents, based on the electrical conductivity response when exposed to halogenated vapors. The influences of the $\mathrm{Si} / \mathrm{Al}$ ratio, zeolite $\mathrm{Y}$ content, and vapor concentration of the halogenated solvents - dichloromethane (DCM), 1,2-dichloroethane (DCE), and chloroform-on the electrical conductivity response of D-PDPA/zeolite Y composites (D-PDPA/Y_H $\mathrm{H}^{+}$) are investigated. The physical adsorption of the halogenated solvents on the composites is examined by FT-IR and UV-Vis spectroscopy.

\section{Experimental}

\subsection{Materials}

Diphenylamine, DPA (reagent, Sigma Aldrich), Ammonium persulfate, $\left(\mathrm{NH}_{4}\right)_{2} \mathrm{~S}_{2} \mathrm{O}_{8}$, (AR grade, Riedel-de Haën), and $37 \%$ v/v of hydrochloric acid, $\mathrm{HCl}$ (ACS reagent, J T Baker), were used at a mole ratio of 100:1 ( $\left.\mathrm{N}_{\mathrm{HCl}} / \mathrm{N}_{\text {monomer }}\right)$. Zeolite $\mathrm{Y}$ (Zeolite International) with $\mathrm{H}^{+}$as a cation $\left(\mathrm{Y}_{-} \mathrm{H}^{+}\right)$, having $\mathrm{Si} / \mathrm{Al}$ ratios of $5.1\left(\mathrm{Y}_{-} \mathrm{H}^{+}[5.1]\right), 30\left(\mathrm{Y}_{-} \mathrm{H}^{+}[30]\right)$, $60\left(\mathrm{Y}_{-} \mathrm{H}^{+}[60]\right)$, and $80\left(\mathrm{Y}_{-} \mathrm{H}^{+}[80]\right)$ were used in powder form. Ammonium hydroxide, $\mathrm{NH}_{4} \mathrm{OH}$ (AR grade, Panreac), toluene (AR grade, Panreac), isopropyl alcohol (AR grade, Burdick \& Jackson), and ethanol (AR grade, Lab Scan) were used without further purification.

\subsection{Chemical vapor detection}

Dichloromethane, DCM (AR grade, Lab Scan), 1,2-dichloroethane, DCE (AR grade, Lab Scan), chloroform (AR grade, Lab Scan), and hexane (AR grade, Lab Scan) were used as the chemical vapors in this work.

\subsection{Synthesis of PDPA and doping process}

Polydiphenylamine (PDPA) was synthesized via chemical oxidative polymerization ${ }^{23,24}$. A DPA solution in toluene was mixed with $\left(\mathrm{NH}_{4}\right)_{2} \mathrm{~S}_{2} \mathrm{O}_{8}$ solution in distilled water. The solution mixture was stirred by using an overhead stirrer, cooled to $0{ }^{\circ} \mathrm{C}$ for 15 minutes, then a $\mathrm{HCl}$ solution in distilled water was added to the mixture. The reaction mixture was continuously stirred at $0{ }^{\circ} \mathrm{C}$ for $4 \mathrm{hr}$; next the green slurry of PDPA was precipitated. The precipitate was washed with isopropyl alcohol at least 3 times. Then the $\mathrm{HCl}$-doped PDPA was dedoped in a mixture of ethanol and ammonium hydroxide solution $(1: 4 \mathrm{v} / \mathrm{v})$ for 24 hours. The neutralized PDPA was doped with $5 \mathrm{M} \mathrm{HCl}$ solution at a mole ratio of 100:1 $\left(\mathrm{N}_{\mathrm{HCl}} / \mathrm{N}_{\text {monomer }}\right)$ and stirred at room temperature for 24 hours. The resultant doped PDPA, D-PDPA, was filtered and dried in a vacuum oven at room temperature for $24 \mathrm{hr}$ to remove excess $\mathrm{HCl}$.

\subsection{Preparation of D-PDPA pellets and D-PDPA/Y_H $H^{+}$composites}

The D-PDPA powder was ground with the $\mathrm{Y}_{-} \mathrm{H}^{+}$powder at various $\mathrm{Y}_{-} \mathrm{H}^{+}$concentrations $(0,5,10,20$, and $30 \% \mathrm{v} / \mathrm{v})$. The D-PDPA powder and D-PDPA/Y_H ${ }^{+}$composites were compressed into pellets for the electrical conductivity and sensitivity measurements with a hydraulic press machine (GRASEBY SPECAC) under a 4-5 ton load. Pellets were produced in disc form using a $1 \mathrm{~cm}$ stainless steel die. The thickness of each pellet was between $0.03-0.04 \mathrm{~cm}$ as measured by a digital thickness gauge (PEAACOCK, dial stand type model PDN-20).

\subsection{Characterization}

Fourier transform infrared (FT-IR) spectra of D-PDPA were recorded with a Thermo Nicolet, Nexus 670 FT-IR spectrometer using a resolution of $2 \mathrm{~cm}^{-1}$, a scan number of 32 , and a wavenumber range of $400-4000 \mathrm{~cm}^{-1}$. Scanning electron micrographs of D-PDPA, Y_H $\mathrm{H}^{+}$, and D-PDPA/Y_H ${ }^{+}$ were taken using a HITACHI, S-4800 scanning electron microscope (SEM) with a magnification of $10000 x$, operating at $10 \mathrm{kV}$. The particle sizes of D-PDPA and $\mathrm{Y} \_\mathrm{H}^{+}$were measured by using a Mastersizer $\mathrm{X}$ particle size analyzer. The surface area and pore size of $\mathrm{Y}_{-} \mathrm{H}^{+}$were measured with a Physisorption, Quantachrome, Autosorb$1 \mathrm{MP}$ surface area analyzer. Zeolite powder was weighed and out gassed at $300{ }^{\circ} \mathrm{C}$ over night before adsorption and desorption with $\mathrm{He}$ and $\mathrm{N}_{2}$ gases. During the operation, the zeolite powder was cooled by liquid $\mathrm{N}_{2}$. The specific density of the D-PDPA and $\mathrm{Y}_{-} \mathrm{H}^{+}$were measured with a pycnometer. The optical properties of the D-PDPA and $\mathrm{Y}_{-} \mathrm{H}^{+}$when exposed to DCM were taken by a UV-Vis spectrophotometer (SHIMADZU, UV 1800) in the wavelength number of $200 \mathrm{~nm}$ to $500 \mathrm{~nm}$ at room temperature.

\subsection{Conductivity and sensitivity measurements}

The conductivity and the sensitivity of D-PDPA, Y_H $\mathrm{H}^{+}$, and D-PDPA/Y_H ${ }^{+}$towards halogenated solvents were measured by a custom built two-point probe connected with a conductivity meter (KEITHLEY 6517A) which applied voltage; the resultant current was measured. The electrical conductivity was calculated by the equation $\sigma=(\mathrm{I} / \mathrm{KVt})$, where $\mathrm{I}$ is the measured current (A), V is the applied voltage $(\mathrm{V})$, $\mathrm{t}$ is the thickness $(\mathrm{cm})$, and $\mathrm{K}$ is the geometric correction factor of the two-point probe as calibrated by standard silicon wafer sheets with known specific resistivity values. All measurements were taken at $27 \pm 1{ }^{\circ} \mathrm{C}$ and at 
atmospheric pressure. The electrical conductivity responses and sensitivities under exposure to air, $\mathrm{N}_{2}$, and halogenated solvents were determined by the equation $\Delta \sigma=\sigma_{\text {halogenated }}$ solvents $-\sigma_{\mathrm{N} 2 \text {, initial }}$ and $\Delta \sigma / \sigma_{\mathrm{N} 2 \text {, initial }}$, respectively, where $\Delta \sigma$ is the difference in the specific electrical conductivity $\left(\mathrm{S} \mathrm{cm}^{-1}\right)$, $\sigma_{\mathrm{N} 2 \text {, initial }}$ is the specific electrical conductivity in $\mathrm{N}_{2}$ before exposure $\left(\mathrm{S} \mathrm{cm}^{-1}\right)$, and $\sigma_{\text {halogenated solvent }}$ is the specific electrical conductivity $\left(\mathrm{S} \mathrm{cm}^{-1}\right)$ under halogenated solvent exposure. The effect of the halogenated solvent concentration was determined at concentrations of 5, 10, 15, 20, 25, 30, 35, and $50 \% \mathrm{v} / \mathrm{v}$ in $\mathrm{N}_{2}$.

\section{Results and Discussion}

\subsection{Characterization of PDPA and $Y_{-} H^{+}$}

The FT-IR spectra of D-PDPA showed absorption peaks at 3388, 3053, 1594, 1505, 1318, 1173, 821, 748, and $748-694 \mathrm{~cm}^{-1}$. These peaks can be assigned to: $\mathrm{N}-\mathrm{H}$ stretching, C-H in the aromatic ring, quinoid ring stretching, phenyl hydrogen, benzenoid ring stretching, vibration band of $\mathrm{N}_{2}$ in quinone, $\mathrm{C}-\mathrm{H}$ out of plane aromatic, 1, 4 substituted on aromatic rings, and C-H out of plane bending vibration, respectively ${ }^{15,24-26}$. The FT-IR spectra of Y_H $\mathrm{H}^{+}$ show major absorption peaks at 1213, 1080, 836, and $459 \mathrm{~cm}^{-1}$. These peaks can be assigned to the asymmetric stretch of the internal tetrahedral, the asymmetric stretch of the external linkage, the symmetric stretch of the external linkage, and the $\mathrm{T}-\mathrm{O}$ bend of the internal tetrahedral, respectively ${ }^{27,28}$. The average particle size of D-PDPA and $\mathrm{Y}_{-} \mathrm{H}^{+}$was $125.96 \pm 5.51 \mu \mathrm{m}$ and $20.66 \pm 0.58 \mu \mathrm{m}$, respectively. The surface area, pore size, and pore volume of $\mathrm{Y}_{-} \mathrm{H}^{+}$are comparable at various $\mathrm{Si} / \mathrm{Al}$ ratios (Table 1 ). The specific density of D-PDPA and $\mathrm{Y}_{-} \mathrm{H}^{+}$is $0.85 \pm 0.01 \mathrm{~g}$ $\mathrm{cm}^{-3}$ and $1.61 \pm 0.35 \mathrm{~g} \mathrm{~cm}^{-3}$, respectively. The micrographs shown in Figure 1 reveal that the shapes of $\mathrm{Y}_{-} \mathrm{H}^{+}$are square

Table 1. Physical characteristics of $\mathrm{Y}_{-} \mathrm{H}^{+}$

\begin{tabular}{cccc}
\hline Si/Al ratio & $\begin{array}{c}\text { Surface area } \\
\left(\mathbf{m}^{2} \mathbf{g}^{-1}\right)\end{array}$ & Pore size $(\mathbf{A})$ & $\begin{array}{c}\text { Pore volume } \\
\left(\mathbf{c m}^{\mathbf{3}} \mathbf{g}^{-\mathbf{~}}\right)\end{array}$ \\
\hline 5.1 & $617.3 \pm 2.27$ & $3.665 \pm 0.01$ & $0.403 \pm 0.01$ \\
30 & $731.0 \pm 2.42$ & $3.670 \pm 0.02$ & $0.569 \pm 0.01$ \\
60 & $688.8 \pm 7.62$ & $3.675 \pm 0.04$ & $0.608 \pm 0.03$ \\
80 & $766.1 \pm 3.25$ & $3.691 \pm 0.01$ & $0.555 \pm 0.08$ \\
\hline
\end{tabular}

shaped (Figure 1a) and the shape of the D-PDPA particles (Figure 1b) is irregular. Nevertheless, the $\mathrm{Y}_{-} \mathrm{H}^{+}$particles are dispersed uniformly within the D-PDPA matrix (Figure 1c).

\subsection{Sensitivity of D-PDPA and $Y \_H^{+}$towards halogenated solvents}

The electrical conductivity sensitivity of D-PDPA, Y_H ${ }^{+}$ towards the four solvents is shown in Figure 2. The D-PDPA and $\mathrm{Y}_{-} \mathrm{H}^{+}$exhibit negative responses towards all four solvents. Table 2 shows the initial electrical conductivity values of D-PDPA, Y_H ${ }^{+}$, D-PDPA/Y_H ${ }^{+}[80]$ when exposed to halogenated solvents. The sensitivity values of D-PDPA, Y_H ${ }^{+}$towards the four solvents, shown in Table 3, show that D-PDPA and $\mathrm{Y}_{-} \mathrm{H}^{+}$possess maximum sensitivity responses towards DCM followed by DCE and chloroform, but they hardly respond to hexane. This is presumably due to the differences in the molecular structure, the strength of the electrostatic interaction, the dipole moment, and the dielectric constant ${ }^{29}$ (Table 4). The interaction between D-PDPA and the vapors occurs at the $\mathrm{N}-\mathrm{H}^{+}$site and the $\mathrm{Cl}^{-}$ atom. Since, the dipole moment of DCM (1.80) is higher than that of DCE (1.60) and chloroform (1.10), the dipole-dipole interaction between the $\mathrm{Cl}^{-}$atom of the DCM molecule and the $\mathrm{N}-\mathrm{H}^{+}$site of D-PDPA is stronger than those of DCE and chloroform $^{30}$. Therefore, the sensitivity of D-PDPA toward DCM is higher than those of other vapors. Because the hexane structure is an aliphatic hydrocarbon chain and it has no dipole moment, the interaction between D-PDPA and

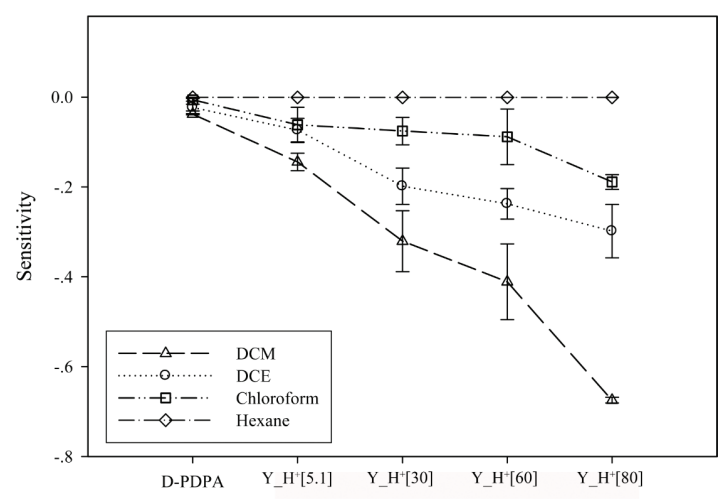

Figure 2. The sensitivity of D-PDPA, $\mathrm{Y}_{-} \mathrm{H}^{+}$at various Si/Al ratios when exposed to halogenated solvents at $27 \pm 1^{\circ} \mathrm{C}$ and $1 \mathrm{~atm}$.

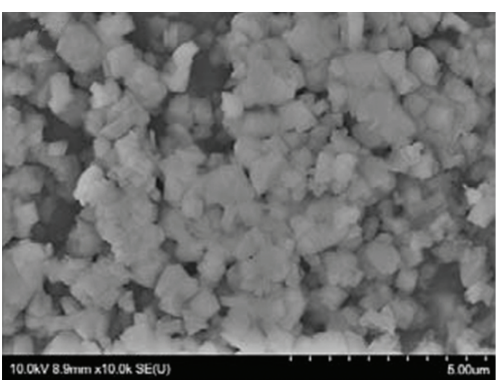

(a)

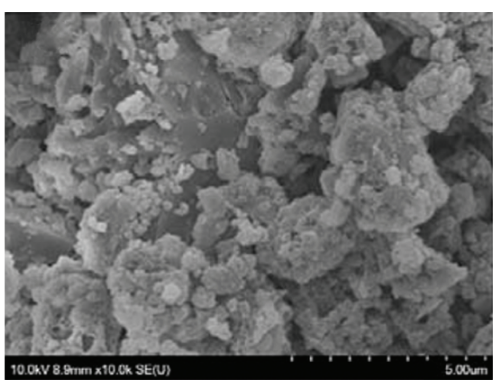

(b)



(c)

Figure 1. SEM micrographs at 10000x of: (a) $\mathrm{Y}_{-} \mathrm{H}^{+}$; (b) D-PDPA; and (c) D-PDPA/Y_H $\mathrm{H}^{+}$. 
Table 2. The initial electrical conductivity of D-PDPA, Y_H+ $\mathrm{H}^{+}$, D-PDPA/Y_ $\mathrm{H}^{+}[80]$ in $\mathrm{N}_{2}$ before exposed to halogenated solvents at $27 \pm 1^{\circ} \mathrm{C}$ and $1 \mathrm{~atm}$.

\begin{tabular}{|c|c|c|c|c|c|}
\hline \multirow{2}{*}{ Solvent Vapor } & \multicolumn{5}{|c|}{ Initial conductivity in $\mathrm{N}_{2}$ before exposed to the solvents, $\sigma_{\mathrm{N} 2}\left(\mathrm{~S} \mathrm{~cm}^{-1}\right)$} \\
\hline & D-PDPA & $\mathbf{Y} \_\mathbf{H}^{+}[5.1]$ & Y_H+[30] & Y_H $H^{+}[60]$ & $\mathbf{Y} \_\mathbf{H}^{+}[\mathbf{8 0}]$ \\
\hline DCM & $(2.29 \pm 0.91) \times 10^{-4}$ & $(6.49 \pm 2.46) \times 10^{-5}$ & $(7.87 \pm 3.45) \times 10^{-5}$ & $(1.31 \pm 0.26) \times 10^{-4}$ & $(2.64 \pm 0.39) \times 10^{-4}$ \\
\hline DCE & $(1.83 \pm 0.45) \times 10^{-4}$ & $(6.41 \pm 0.76) \times 10^{-5}$ & $(6.98 \pm 0.66) \times 10^{-5}$ & $(7.49 \pm 1.63) \times 10^{-5}$ & $(9.34 \pm 1.05) \times 10^{-5}$ \\
\hline Chloroform & $(1.34 \pm 0.01) \times 10^{-4}$ & $(9.69 \pm 1.15) \times 10^{-5}$ & $(8.92 \pm 0.39) \times 10^{-5}$ & $(9.26 \pm 0.05) \times 10^{-5}$ & $(1.44 \pm 0.68) \times 10^{-4}$ \\
\hline Hexane & $(1.42 \pm 0.16) \times 10^{-4}$ & $(6.86 \pm 0.03) \times 10^{-5}$ & $(8.66 \pm 0.81) \times 10^{-5}$ & $(1.04 \pm 0.06) \times 10^{-4}$ & $(1.75 \pm 0.02) \times 10^{-4}$ \\
\hline Solvent Vapor & $\begin{array}{c}\text { D-PDPA/5\% } \mathrm{Y}_{-} \\
\mathrm{H}^{+}[\mathbf{8 0}]\end{array}$ & $\begin{array}{c}\text { D-PDPA } / 10 \% \\
\text { Y_H } \mathbf{H}^{+}[\mathbf{8 0}] \\
\end{array}$ & $\begin{array}{c}\text { D-PDPA/20\% } \\
\text { Y_H } \mathbf{H}^{+}[80]\end{array}$ & $\begin{array}{c}\text { D-PDPA/30\% } \\
\text { Y__ } \mathbf{H}^{+}[\mathbf{8 0}] \\
\end{array}$ & \\
\hline DCM & $(1.60 \pm 0.35) \times 10^{-4}$ & $(1.39 \pm 0.07) \times 10^{-4}$ & $(1.97 \pm 0.22) \times 10^{-4}$ & $(2.24 \pm 0.04) \times 10^{-4}$ & \\
\hline DCE & $(2.15 \pm 0.31) \times 10^{-4}$ & $(2.26 \pm 0.24) \times 10^{-4}$ & $(1.95 \pm 0.25) \times 10^{-4}$ & $(1.90 \pm 0.10) \times 10^{-4}$ & \\
\hline Chloroform & $(1.70 \pm 0.01) \times 10^{-4}$ & $(1.75 \pm 0.14) \times 10^{-4}$ & $(3.08 \pm 1.88) \times 10^{-4}$ & $(2.11 \pm 1.03) \times 10^{-4}$ & \\
\hline Hexane & $(1.61 \pm 0.02) \times 10^{-4}$ & $(1.41 \pm 0.15) \times 10^{-4}$ & $(1.73 \pm 0.07) \times 10^{-4}$ & $(1.34 \pm 0.29) \times 10^{-4}$ & \\
\hline
\end{tabular}

Table 3. Electrical conductivity sensitivity of D-PDPA, Y_H+, D-PDPA/Y_H+[80] when exposed to halogenated and non-halogenated solvents at $27 \pm 1^{\circ} \mathrm{C}$ and $1 \mathrm{~atm}$.

\begin{tabular}{|c|c|c|c|c|}
\hline \multirow{2}{*}{ Material } & \multicolumn{4}{|c|}{ Response $\left(\Delta \sigma=\sigma \text { halogenated solvent }-\sigma_{\mathrm{N} 2}\right)^{\mathrm{a}}$} \\
\hline & DCM & DCE & chlroform & hexane \\
\hline D-PDPA & $(-8.77 \pm 3.82) \times 10^{-6}$ & $(-1.79 \pm 0.73) \times 10^{-5}$ & $(-8.07 \pm 4.05) \times 10^{-7}$ & $(-5.11 \pm 3.25) \times 10^{-8}$ \\
\hline $\mathrm{Y}_{-} \mathrm{H}^{+}[5.1]$ & $(-9.60 \pm 4.81) \times 10^{-6}$ & $(-4.81 \pm 2.25) \times 10^{-6}$ & $(-5.76 \pm 3.09) \times 10^{-6}$ & $(-4.30 \pm 0.56) \times 10^{-8}$ \\
\hline Y_H+[30] & $(-2.10 \pm 2.41) \times 10^{-5}$ & $(-1.32 \pm 0.52) \times 10^{-5}$ & $(-6.80 \pm 3.01) \times 10^{-6}$ & $(-5.75 \pm 2.70) \times 10^{-8}$ \\
\hline Y_H $\mathrm{H}^{+}[60]$ & $(-5.28 \pm 0.03) \times 10^{-5}$ & $(-1.78 \pm 0.09) \times 10^{-5}$ & $(-8.20 \pm 5.77) \times 10^{-6}$ & $(-6.61 \pm 2.27) \times 10^{-8}$ \\
\hline $\mathrm{Y}_{-} \mathrm{H}^{+}[80]$ & $(-1.78 \pm 0.25) \times 10^{-4}$ & $(-2.39 \pm 1.48) \times 10^{-5}$ & $(-3.74 \pm 2.87) \times 10^{-5}$ & $(-1.35 \pm 0.35) \times 10^{-7}$ \\
\hline D-PDPA/5\% Y_H+[80] & $(-1.86 \pm 0.28) \times 10^{-5}$ & $(-5.96 \pm 7.78) \times 10^{-6}$ & $(-1.58 \pm 0.02) \times 10^{-6}$ & $(-5.94 \pm 5.92) \times 10^{-8}$ \\
\hline D-PDPA/10\% Y_H+[80] & $(-2.32 \pm 0.11) \times 10^{-5}$ & $(-7.83 \pm 5.74) \times 10^{-6}$ & $(-9.36 \pm 2.31) \times 10^{-7}$ & $(-4.86 \pm 0.88) \times 10^{-8}$ \\
\hline D-PDPA/20\% Y_H+[80] & $(-3.63 \pm 2.35) \times 10^{-5}$ & $(-9.66 \pm 9.04) \times 10^{-6}$ & $(-8.90 \pm 1.84) \times 10^{-6}$ & $(-6.07 \pm 2.91) \times 10^{-8}$ \\
\hline D-PDPA/30\% Y_H+[80] & $(-7.78 \pm 3.79) \times 10^{-5}$ & $(-3.37 \pm 0.26) \times 10^{-5}$ & $(-8.32 \pm 0.62) \times 10^{-6}$ & $(-5.10 \pm 0.50) \times 10^{-8}$ \\
\hline \multirow{2}{*}{ Material } & \multicolumn{4}{|c|}{ Sensitivity $\left(\Delta \sigma / \sigma_{\mathrm{N} 2}\right)^{\mathrm{b}}$} \\
\hline & DCM & DCE & chlroform & hexane \\
\hline D-PDPA & $(-3.83 \pm 0.15) \times 10^{-2}$ & $(-2.42 \pm 0.73) \times 10^{-2}$ & $(-6.01 \pm 3.02) \times 10^{-3}$ & $(-3.60 \pm 2.70) \times 10^{-4}$ \\
\hline $\mathrm{Y}_{-} \mathrm{H}^{+}[5.1]$ & $(-1.48 \pm 0.19) \times 10^{-1}$ & $(-7.50 \pm 2.64) \times 10^{-2}$ & $(-5.94 \pm 3.92) \times 10^{-2}$ & $(-6.27 \pm 0.79) \times 10^{-4}$ \\
\hline $\mathrm{Y}_{-} \mathrm{H}^{+}[30]$ & $(-2.67 \pm 2.09) \times 10^{-1}$ & $(-1.89 \pm 0.06) \times 10^{-1}$ & $(-7.63 \pm 3.04) \times 10^{-2}$ & $(-6.63 \pm 2.50) \times 10^{-4}$ \\
\hline $\mathrm{Y}_{-} \mathrm{H}^{+}[60]$ & $(-4.03 \pm 0.84) \times 10^{-1}$ & $(-2.34 \pm 0.03) \times 10^{-1}$ & $(-8.86 \pm 6.18) \times 10^{-2}$ & $(-6.35 \pm 2.54) \times 10^{-4}$ \\
\hline $\mathrm{Y}_{-} \mathrm{H}^{+}[80]$ & $(-6.74 \pm 0.06) \times 10^{-1}$ & $(-2.56 \pm 1.30) \times 10^{-1}$ & $(-2.60 \pm 0.87) \times 10^{-1}$ & $(-7.76 \pm 1.90) \times 10^{-4}$ \\
\hline D-PDPA/5\% Y_H+[80] & $(-1.16 \pm 0.08) \times 10^{-1}$ & $(-2.90 \pm 0.03) \times 10^{-2}$ & $(-9.27 \pm 0.77) \times 10^{-3}$ & $(-3.69 \pm 3.68) \times 10^{-4}$ \\
\hline D-PDPA/10\% Y_H+[80] & $(-1.67 \pm 0.16) \times 10^{-1}$ & $(-3.50 \pm 0.03) \times 10^{-2}$ & $(-5.36 \pm 1.28) \times 10^{-3}$ & $(-3.44 \pm 0.99) \times 10^{-4}$ \\
\hline D-PDPA/20\% Y_H+[80] & $(-1.85 \pm 0.99) \times 10^{-1}$ & $(-5.00 \pm 4.03) \times 10^{-2}$ & $(-2.89 \pm 1.43) \times 10^{-2}$ & $(-3.51 \pm 0.25) \times 10^{-4}$ \\
\hline D-PDPA/30\% Y_H+[80] & $(-3.48 \pm 1.64) \times 10^{-1}$ & $(-1.77 \pm 0.04) \times 10^{-1}$ & $(-3.95 \pm 1.86) \times 10^{-2}$ & $(-3.79 \pm 0.44) \times 10^{-4}$ \\
\hline
\end{tabular}

${ }^{a} \Delta \sigma=$ the electrical response; ${ }^{\mathrm{b}} \Delta \sigma / \sigma_{\mathrm{N} 2}=$ electrical conductivity sensitivity.

hexane vapor is suspected to be physical adsorption on the matrix surface without the dipole-dipole interaction, unlike the other chlorinated hydrocarbon molecules ${ }^{31-33}$. Therefore, the sensitivity of D-PDPA towards hexane is low. For zeolite, the interaction between $\mathrm{Y}_{-} \mathrm{H}^{+}$and the vapors is the hydrogen bonding at the $\mathrm{Si}_{-} \mathrm{O}^{-}$or $\mathrm{Al}_{-}-\mathrm{O}^{-}$site and the $-\mathrm{H}$ atom of the vapor molecules. The sensitivity of $\mathrm{Y}_{-} \mathrm{H}^{+}$towards DCM is also higher than those of DCE $(\sim 50 \%)$ and chloroform $(\sim 60 \%)$. For DCM, there are two hydrogen atoms that form the hydrogen bond with D-PDPA and $\mathrm{Y}_{-} \mathrm{H}^{+}$. For DCE, a lower sensitivity than DCM occurs due to the larger chemical structure of DCE over DCM, known as the steric effect. For chloroform, since there is only one hydrogen atom in the chemical structure, and the dipole moment and dielectric constant are also very low, the strength of the hydrogen bonding between $\mathrm{Y}_{-} \mathrm{H}^{+}$and chloroform is lower than the strength of the hydrogen bonding between $\mathrm{Y}_{-} \mathrm{H}^{+}$and DCM and $\mathrm{Y}_{-} \mathrm{H}^{+}$and $\mathrm{DCE}^{30}$. Moreover, the sensitivity of $\mathrm{Y}_{-} \mathrm{H}^{+}$ increases with increasing $\mathrm{Si} / \mathrm{Al}$ ratio. This occurs because the hydrophobicity of zeolite increases with the $\mathrm{Si} / \mathrm{Al}$ ratio $^{34}$. Hence, the halogenated solvents, which are non-polar solvents, produce more favorable responses towards the zeolites $\mathrm{Y}_{-} \mathrm{H}^{+}$with higher $\mathrm{Si} / \mathrm{Al}$ ratios. Therefore, the zeolite $\mathrm{Y}_{-} \mathrm{H}^{+}[80]$ was chosen to form composites with D-PDPA, in order to further improve the sensitivity of D-PDPA towards the halogenated solvents. 


\subsection{Sensitivity of D-PDPA/Y_H $H^{+}[80]$ composites toward halogenated solvents: Effect of zeolite $Y$ contents}

The effect of $\mathrm{Y}_{-} \mathrm{H}^{+}[80]$ content on the sensitivity of D-PDPA/Y_H $\mathrm{H}^{+}[80]$ towards the halogenated solvents was investigated. The composites show negative sensitivity towards the solvents (Table 3 ). According to the doping theory ${ }^{35}$, D-PDPA is doped via the $\mathrm{p}$ type doping process. When it is exposed to the vapors, which are electron-donating groups, the vapors will give up an electron to fill the hole on the D-PDPA backbone. Thus, the electron mobility along the D-PDPA backbone is more difficult, resulting in a decrease in conductivity, which is why the composites show negative sensitivity towards the solvents. It appears that the sensitivities of the composites are higher than those of pure D-PDPA by about 1 order of magnitude towards DCM and DCE; the sensitivity increases with increasing $\mathrm{Y}_{-} \mathrm{H}^{+}[80]$ content (Figure 3). With a higher zeolite content, a greater interaction results between the micro-porous structure and the target

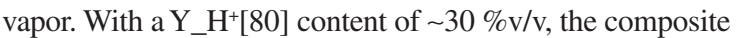
shows the highest sensitivity. The sensitivity of chloroform is quite constant with respect to the $\mathrm{Y}_{-} \mathrm{H}^{+}[80]$ content because it has no dipole moment and very low dielectric constants ${ }^{36}$. The composite does not respond to hexane, even when the zeolite content increases to $30 \% \mathrm{v} / \mathrm{v}$, because the interaction between the composite and hexane is only physical absorption. The selectivity of the composite towards the three halogenated solvents is in this order: DCM $>$ DCE $>$ chloroform. Therefore, it can be concluded that the addition of zeolites can improve the sensitivity of D-PDPA and it can discriminate a nonhalogenated solvent from halogenated solvents.

Table 4. Values of dielectric constant $(\varepsilon)$, dipole moment $(\mu)$ of the solvent tested ${ }^{29}$

\begin{tabular}{lcc}
\hline \multicolumn{1}{c}{ Solvents } & Dipole moment & Dielectric constant \\
\hline Dichloromethane & 1.80 & 9.10 \\
1,2 dichloroethane & 1.60 & 10.45 \\
chloroform & 1.10 & 4.80 \\
hexane & 0.00 & 1.88 \\
\hline
\end{tabular}

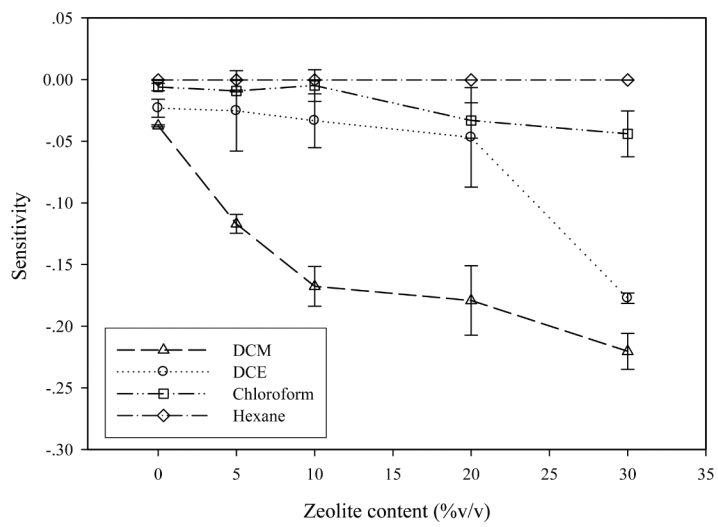

Figure 3. The sensitivity of D-PDPA/Y_ $\mathrm{H}^{+}[80]$ at various zeolite concentrations when exposed to halogenated solvents at $27 \pm 1^{\circ} \mathrm{C}$ and $1 \mathrm{~atm}$.

\subsection{Sensitivity of D-PDPA/Y_H $H^{+}[80]$ composites toward halogenated solvents: Effect of vapor concentration}

The composite consisting of $30 \% \mathrm{v} / \mathrm{v}$ of $\mathrm{Y}_{-} \mathrm{H}^{+}[80]$ with D-PDPA (D-PDPA/30\% Y_H ${ }^{+}[80]$ ) was next investigated with respect to DCM and DCE at various vapor concentrations. The sensitivity of the composite, which is exposed to DCM, increases from $(-1.23 \pm 0.004) \times 10^{-2}$ to $(-3.47 \pm 1.64) \times 10^{-1}$ as the vapor concentration increases from 7,696 ppm to $153,914 \mathrm{ppm}$, respectively (Figure 4). When the composite is exposed to DCE, the sensitivity increases from $(-1.09 \pm 0.96) \times 10^{-1}$ to $(-1.77 \pm 0.04) \times 10^{-1}$ as the vapor concentration increases from 1,015 ppm to 20,299 ppm, respectively. When the composites are exposed to both of DCM and DCE, they exhibit linear relationships between the sensitivity and vapor concentration. As the vapor concentration increases, the sensitivity of D-PDPA/30\% Y_H $\mathrm{H}^{+}[80]$ increases. A similar result has been also found by Jiang et al. ${ }^{37}$. They prepared a polypyrrole and polyvinylalcohol composite via in situ vapor state polymerization to detect methanol vapors. The composite showed a linear relationship between sensitivity and gas concentration in the gas range of 50-1059 ppm. The minimum concentrations of DCM and DCE that the composite can detect are $7696 \mathrm{ppm}$ and $1015 \mathrm{ppm}$, respectively; these values are equivalent to the $\mathrm{IDLHs}^{30,38,39}$. The chemical interactions between DCM and the composites were investigated by FT-IR spectroscopy and UV-VIS spectroscopy. Figure 5 shows the FT-IR spectra of the vapors. Before exposure to DCM, the absorption peaks at $1594,1505,1318,1213,1080,836$, and $748 \mathrm{~cm}^{-1}$ can be assigned to the quinoid ring stretching of D-PDPA ${ }^{25}$, the phenyl hydrogen of D-PDPA ${ }^{15}$, the benzenoid ring stretching of D-PDPA, the asymmetric stretch of the internal tetrahedral in $\mathrm{Y}_{-} \mathrm{H}^{+}[80]$, the asymmetric stretch of the external linkage, the symmetric stretch of external linkage ${ }^{27,28}$, and the 1,4 substituted on aromatic rings ${ }^{24}$, respectively. During exposure to DCM, new peaks occur at 3097, 1285, and $752 \mathrm{~cm}^{-1}$ which can be assigned to the hydrogen bonding between the oxygen atom of $\mathrm{Y}_{-} \mathrm{H}^{+}$and the hydrogen atom

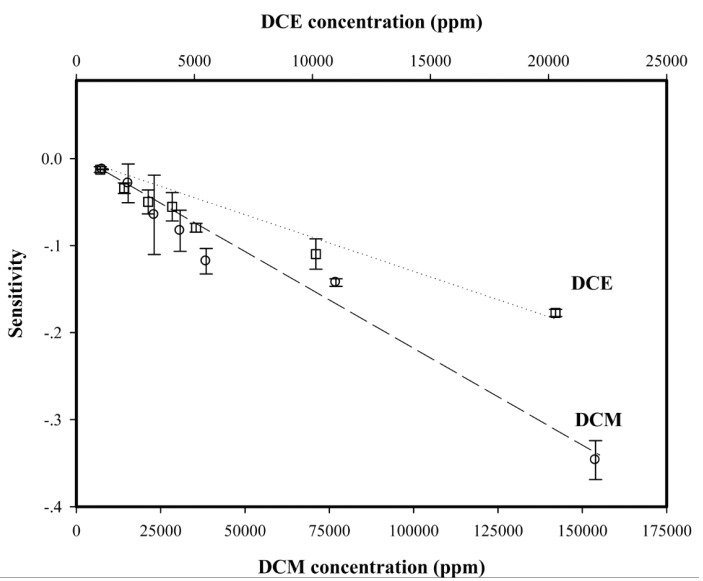

Figure 4. The sensitivity of D-PDPA/30\% Y_ $\mathrm{H}^{+}[80]$ toward DCM and DCE at different concentrations at $27 \pm 1^{\circ} \mathrm{C}$ and $1 \mathrm{~atm}$. 


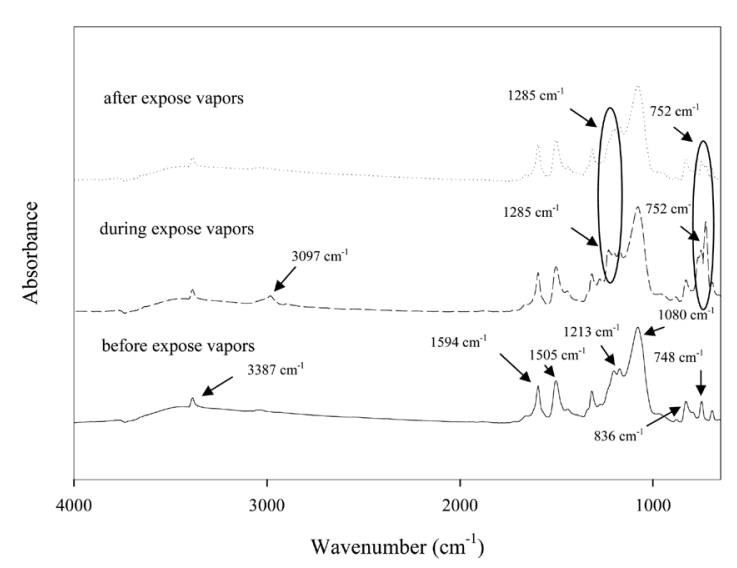

Figure 5. FT-IR spectra of D-PDPA/30\% Y_H $\mathrm{H}^{+}[80]$ when exposed to DCM and DCE vapors at $27 \pm 1^{\circ} \mathrm{C}$ and $1 \mathrm{~atm}$.

of DCM molecule ${ }^{40}, \mathrm{CH}_{2}$ in $\mathrm{CH}_{2}-\mathrm{Cl}^{41}$, and the interaction of the chlorine atom of DCM and the nitrogen atom of D-PDPA backbone ${ }^{42}$, respectively. After exposure to DCM, the peaks at 3097,1285 , and $752 \mathrm{~cm}^{-1}$ disappear. This indicates that no interaction occurs between the composite and DCM molecules. The proposed mechanism is shown in Figure 6. From the proposed mechanism, the FT-IR absorption band of D-PDPA cannot be clearly discerned when exposed to DCM, thus the second derivative of the spectrum is used instead. During exposure to DCM, the absorption bands at 1596 and $1318 \mathrm{~cm}^{-1}$ corresponding to the quinoid ring stretching and the benzenoid ring stretching, respectively shift from the original positions before exposed to DCM by $4 \mathrm{~cm}^{-1}$ for thequinoid ring stretching and by $7 \mathrm{~cm}^{-1}$ for the benzenoid ring stretching as shown in Figure 7. Anitha and Subramanian ${ }^{43}$ have also observed that the FT-IR absorption band of polyaniline when exposed to DCM shifted by $3 \mathrm{~cm}^{-1}$ due to the electron displacement from chlorine atom of DCM to the polyaniline backbone.

The UV-Vis spectra are used to confirm the interaction between DCM and the composite. The spectrum of the D-PDPA is shown in Figure 8a. There are two absorption bands, one at $282 \mathrm{~nm}$ which corresponds to the characteristic absorption bands of DCM, and the other at $342 \mathrm{~nm}$ which corresponds to the $n-\pi *$ transition at $-\mathrm{N}^{+}=$of the D-PDPA backbone ${ }^{44}$. It is clear from Figure $8 \mathrm{a}$ that the absorption band is blue shifted from $342 \mathrm{~nm}$ to $334 \mathrm{~nm}$ and the absorbance intensity decreases with increasing DCM concentration from $5 \% \mathrm{v} / \mathrm{v}$ to $25 \% \mathrm{v} / \mathrm{v}$. This indicates that the shift in the absorption band is due to DCM giving up an electron to fill hole on D-PDPA back bone. Thus, the electron mobility along the D-PDPA backbone becomes more difficult, and the band gap of D-PDPA increases. It needs more energy to accelerate the electron to a higher conduction band, resulting in the shift of the electronic band of D-PDPA to a higher energy or a lower wavelength ${ }^{16,45}$. The absorption band observed at $271 \mathrm{~nm}$ corresponds to the $-\mathrm{Si}_{-} \mathrm{O}_{4}{ }^{2-}$ site on the $\mathrm{Y}_{-} \mathrm{H}^{+}$framework $^{46}$ (Figure $8 \mathrm{~b}$ ). The absorption band is red shifted from $271 \mathrm{~nm}$ to $277 \mathrm{~nm}$ and the absorbance intensity decreases with increasing DCM concentration from $5 \% \mathrm{v} / \mathrm{v}$ to $25 \% \mathrm{v} / \mathrm{v}$ because the electron

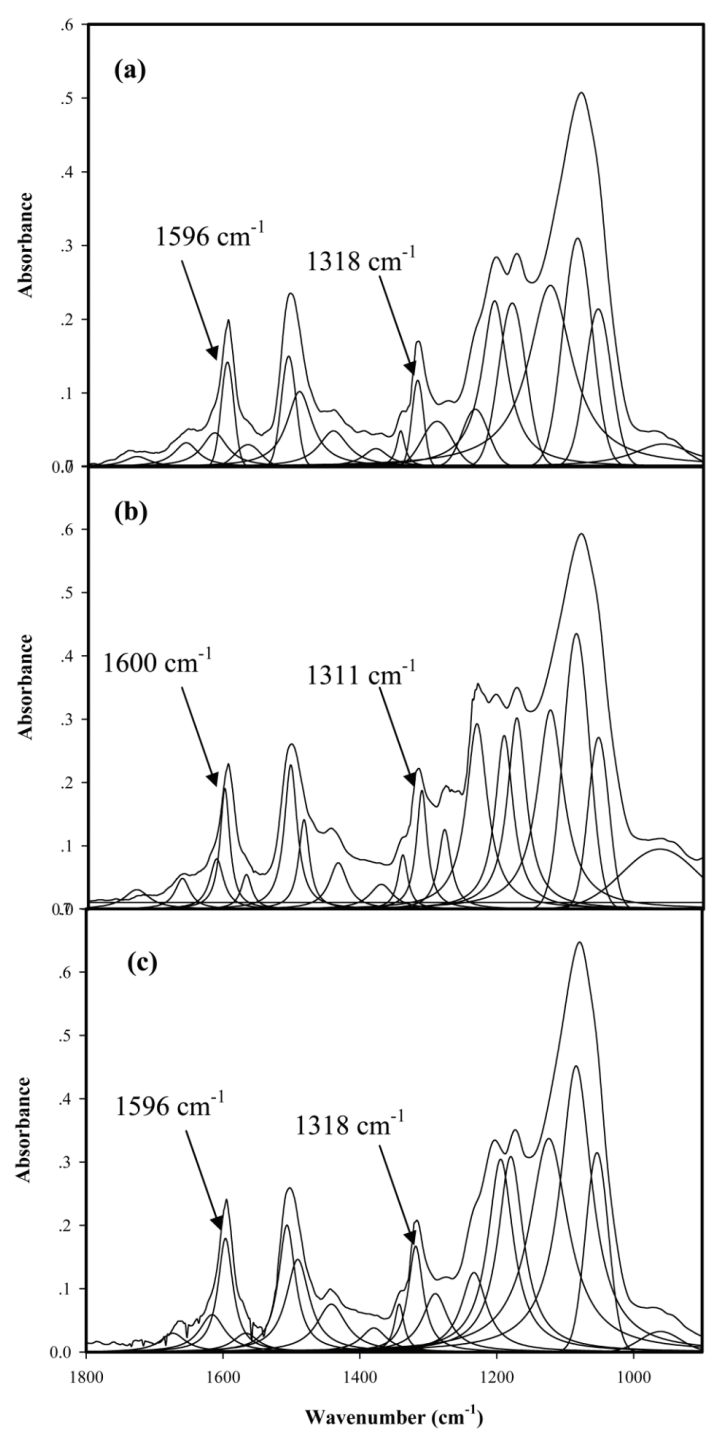

Figure 6. FT-IR second derivative of D-PDPA/30\%Y_ $\mathrm{H}^{+}[80]$ composite when exposed to DCM.

donor and acceptor interact between the $-\mathrm{Si}_{-} \mathrm{O}_{4}{ }^{2-}$ site of the $\mathrm{Y} \_\mathrm{H}^{+}$framework and the $-\mathrm{H}$ atom of the DCM molecule. For D-PDPA/30\% Y_H $\mathrm{H}^{+}[80]$ composite (Figure 8c), they are also two absorption bands at $\sim 340 \mathrm{~nm}$ and $\sim 265 \mathrm{~nm}$ which correspond to the $n-\pi^{*}$ transition at $-\mathrm{N}^{+}=$of the D-PDPA backbone and the $-\mathrm{Si}_{-} \mathrm{O}_{4}{ }^{2-}$ site on the $\mathrm{Y}_{-} \mathrm{H}^{+}$framework, respectively. The absorption band at $342 \mathrm{~nm}$ is blue shifted from $340 \mathrm{~nm}$ to $336 \mathrm{~nm}$. The absorption band at $262 \mathrm{~nm}$ is red shifted to $275 \mathrm{~nm}$. This occurs similarly to D-PDPA and $\mathrm{Y} \_\mathrm{H}^{+}[80]$. It can be concluded that the interaction between the composite of D-PDPA/30\% Y_H $\mathrm{H}^{+}[80]$ and DCM occurs as shown in the proposed mechanism (Figure 7). Choi and co-worker ${ }^{47}$ studied iodine absorption on various zeolites. The absorption band was gradually red shifted when the iodine molecules increasingly absorbed on the zeolite $\mathrm{Y}$ framework. They attributed the phenomenon to $-\mathrm{Si}_{-} \mathrm{O}_{4}{ }^{2-}$ donating an electron to the adsorbed iodine molecules. 
<smiles></smiles><smiles>CO[Si](C)(O)O[Si](C)(O[Si])O[Si](O)(O)O[Si](O)(O)O[Si](O)(O)O[Si](O)(O)O</smiles><smiles>ClCCl</smiles><smiles>Cc1ccc(N=C2C=CC(=c3ccc(=C4C=CC(=[NH+]c5ccc(C)cc5)C=C4)cc3)C=C2)cc1</smiles>

Figure 7. Proposed mechanism of adsorbed DCM, and DCE on D-PDPA/30\% Y_H $\mathrm{H}^{+}[80]$.

\subsection{The temporal response $D-P D P A / Y \_H^{+}[80]$ composites}

The induction time of the D-PDPA when exposed to DCM, DCE, and chloroform was around $13.50 \pm 2.12$, $8.56 \pm 1.25,15.00 \pm 1.41 \mathrm{~min}$, respectively. For the D-PDPA/30\% Y_H ${ }^{+}[80]$ composite, the induction time was about $18.50 \pm 0.71 \mathrm{~min}$ for DCM, $17.65 \pm 0.43 \mathrm{~min}$ for DCE, $15.95 \pm 1.34$ min for chloroform. The results showed that the induction time of the composite was higher than D-PDPA. This suggests that the presence of zeolite, a micro porous structure, introduces more active sites for the vapors to absorb. D-PDPA showed a reduction time when exposed to DCM, DCE, and chloroform of $9.50 \pm 4.86$, $6.35 \pm 1.57$, and $13.75 \pm 7.84 \mathrm{~min}$, respectively. The reduction times of the composite when exposed to DCM, DCE, and chloroform were $6.00 \pm 3.04,6.10 \pm 0.49$, and $7.05 \pm 2.87 \mathrm{~min}$, respectively. The reduction time of the D-PDPA/30\% Y_H $\mathrm{H}^{+}[80]$ composite was also higher than the D-PDPA due to the micro porous structure of the zeolite. A similar result was also found by Thuwachaowsoan and co-worker ${ }^{48}$. They fabricated a composites of poly(3-thiopheneacetic acid) (Pth) with zeolites $\mathrm{L}$, mordenite (MOR), and beta (BETA) as $\mathrm{H}_{2}$ gas sensors. They found that the induction time of the Pth 200:1/BETA 


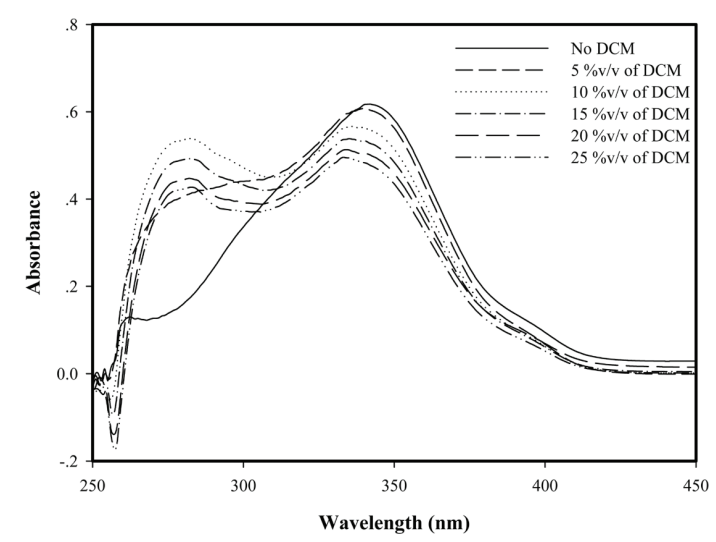

(a)

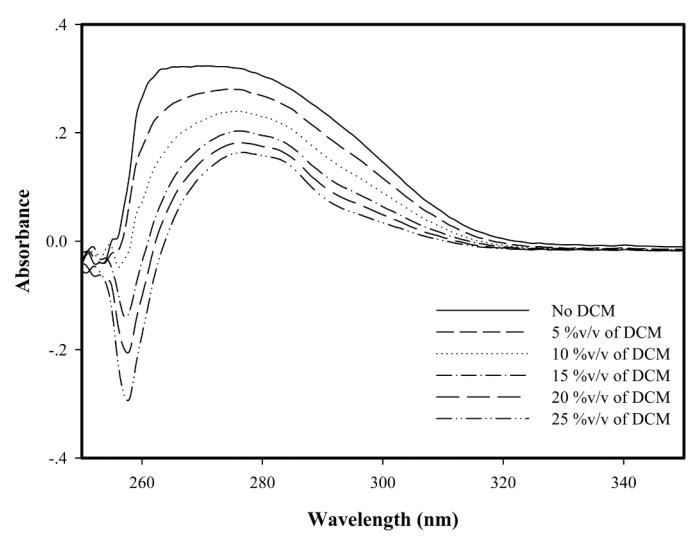

(b)

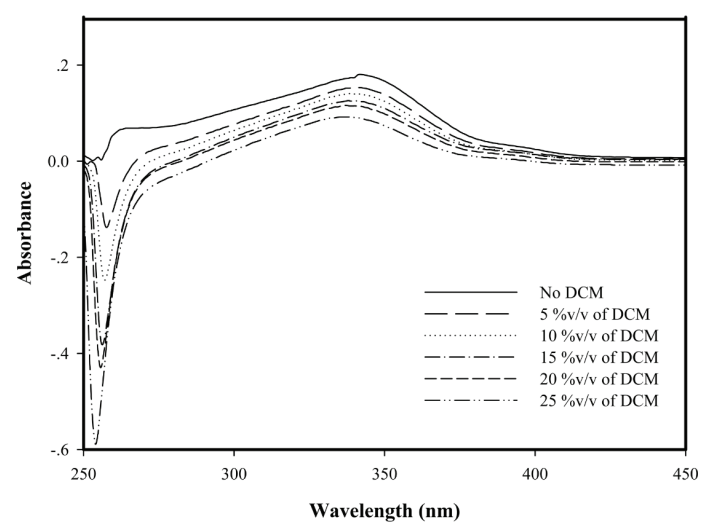

(c)

Figure 8. UV-Vis spectra of: a) D-PDPA; b) $\mathrm{Y}_{-} \mathrm{H}^{+}$when exposed to DCM; and c) D-PDPA/30\% Y_H $\mathrm{H}^{+}[80]$ composite at various concentrations.

20 was longer than that of the polymer and other composites. They concluded that the presence of zeolite introduces more reactive sites to interact with the $\mathrm{H}_{2}$ molecules.

\section{Conclusions}

D-PDPA was fabricated into a composite with $\mathrm{Y}_{-} \mathrm{H}^{+}$to detect the toxic halogenated solvent vapors DCM, DCE, chloroform, and hexane. The composites show improved sensitivity responses towards DCM, DCE, and chloroform than hexane, because of the differences in the chemical structures of the dipole moment, and the dielectric constants of the target vapors. The sensitivities of the composites increase with increasing $\mathrm{Si} / \mathrm{Al}$ ratio, zeolite content, and vapor concentration due to greater interactions between the zeolite and target vapors. For the temporal response, the induction and recovery times of the composite were higher than those of the pristine D-PDPA due to more active sites being available for the vapor molecules. The UV-Vis spectra show that the interaction between the composite and DCM does exist; the UV absorption band of D-PDPA and $\mathrm{Y}_{-} \mathrm{H}^{+}$ is shifted by the delocalization of the $\mathrm{Cl}^{-}$atom. The electron donor, acceptor interaction occurs between $\mathrm{Y}_{-} \mathrm{H}+$ and the $-\mathrm{H}$ atom when exposed to DCM. It may be concluded that the fabricated composites possess a potential use as a sensing material for discriminating non-halogenated solvents from halogenated hydrocarbon solvents.

\section{Acknowledgements}

The authors would like to acknowledge the financial support from: the Conductive and Electroactive Polymers Research Unit of Chulalongkorn University; the Thailand Research Fund (TRF-RTA); the Royal Thai Government; and the Thailand Graduate Institute of Science and Technology (TGIST) (TGIST-01-54-011). 


\section{References}

1. Sidebottom $\mathrm{H}$ and Franklin J. The atmospheric fate and impact of hydrochlorofluorocarbons and chlorinated solvents. Pure and Apply Chemical. 1996; 68(9):1757-69. http://dx.doi. org/10.1351/pac199668091757

2. Penza M and Cassano G. Application of principal component analysis and artificial neural networks to recognize the individual VOCs of methanol/2-propanol in a binary mixture by SAW multi-sensor array. Sensors and Actuators B: Chemical. 2003; 89(3):269-84. http://dx.doi.org/10.1016/ S0925-4005(03)00002-9

3. Kukla AL, Pavluchenko AS, Shirshov YM, Konoshchuk NV and Posudievsky OY. Application of sensor arrays based on thin films of conducting polymers for chemical recognition of volatile organic solvents. Sensors and Actuators B: Chemical. 2009; 135(2):541-51. http://dx.doi.org/10.1016/j. snb.2008.09.027

4. Feng $\mathbf{J}$ and MacDiarmid AG. Sensors using octaaniline for volatile organic compounds. Synthetic Metals. 1999; 102(13):1304-5. http://dx.doi.org/10.1016/S0379-6779(98)00989-8

5. Whitaker AM and Jones CS. Report of 1500 Chloroform Anesthetics Administered With a Precision Vaporizer*. Anesthesia \& Analgesia. 1965; 44(1):60-5. http://dx.doi. org/10.1213/00000539-196501000-00016

6. NOAA's Office of Response and Restoration (OR\&R). Immediately Dangerous to Life and Health Limits (IDLHs). Available from: <http://response.restoration.noaa.gov/idlhs $>$.

7. Featherstone HW. Chloroform. Anesthesiology. 1947; 8(4):362-71. http://dx.doi.org/10.1097/00000542-194707000-00004

8. Anesthesia. Available from: <http://en.wikipedia.org/w/index. php?title $=$ Anesthesia\&oldid $=515002047>$.

9. Williams PL, James RC and Roberts SM. Principles of Toxicology: Environmental and Industrial Applications. Wiley; 2000.

10. Bailey ALPS, Pisanelli AM and Persaud KC. Development of conducting polymer sensor arrays for wound monitoring. Sensors and Actuators B: Chemical. 2008; 131(1):5-9. http:// dx.doi.org/10.1016/j.snb.2007.12.035

11. Bai H and Shi G. Gas Sensors Based on Conducting Polymers Sensors. 2007; 7(3):267-307. http://dx.doi.org/10.3390/ s7030267

12. Albert KJ, Lewis NS, Schauer CL, Sotzing GA, Stitzel SE, Vaid TP et al. Cross-Reactive Chemical Sensor Arrays. Chemical Reviews. 2000; 100(7):2595-626. http://dx.doi.org/10.1021/ cr980102w

13. Li CY, Wen TC, Guo TF and Hou SS. A facile synthesis of sulfonated poly(diphenylamine) and the application as a novel hole injection layer in polymer light emitting diodes. Polymer. 2008; 49(4):957-64. http://dx.doi.org/10.1016/j. polymer.2007.12.031

14. Hua F and Ruckenstein E. Hyperbranched Sulfonated Polydiphenylamine as a Novel Self-Doped Conducting Polymer and Its pH Response. Macromolecules. 2005; 38(3):888-98. http://dx.doi.org/10.1021/ma047756t

15. HuaF and RuckensteinE. Water-Soluble Conducting Poly(ethylene oxide)-Grafted Polydiphenylamine Synthesis through a "Graft Onto" Process. Macromolecules. 2003; 36(26):9971-8. http:// dx.doi.org/10.1021/ma030431c

16. Chung CY, Wen TC and Gopalan A. Identification of electrochromic sites in poly(diphenylamine) using a novel absorbance-potential-wavelength profile. Electrochimica Acta. 2001; 47(3):423-31. http://dx.doi.org/10.1016/S0013$4686(01) 00742-3$
17. Santhosh P, Manesh KM, Uthayakumar S, Gopalan AI and Lee KP. Hollow spherical nanostructured polydiphenylamine for direct electrochemistry and glucose biosensor. Biosensors and Bioelectronics. 2009; 24(7):2008-14. http://dx.doi. org/10.1016/j.bios.2008.10.004

18. Santhosh P, Manesh KM, Gopalan A and Lee KP. Novel amperometric carbon monoxide sensor based on multi-wall carbon nanotubes grafted with polydiphenylamine - Fabrication and performance. Sensors and Actuators $B$ : Chemical. 2007; 125(1):92-9. http://dx.doi.org/10.1016/j. snb.2007.01.044

19. Rodríguez Presa MJ, Posadas D and Florit MI. Conditioning treatment to improve the potentiometric $\mathrm{pH}$ response of polydiphenylamine modified electrodes. Sensors and Actuators B: Chemical. 2007; 123(1):142-7. http://dx.doi.org/10.1016/j. snb.2006.08.002

20. Hugon O, Sauvan M, Benech P, Pijolat $C$ and Lefebvre F. Gas separation with a zeolite filter, application to the selectivity enhancement of chemical sensors. Sensors and Actuators B: Chemical. 2000; 67(3):235-43. http://dx.doi.org/10.1016/ S0925-4005(00)00514-1

21. Wu JY, Liu QL, Xiong Y, Zhu AM and Chen Y. Molecular Simulation of Water/Alcohol Mixtures' Adsorption and Diffusion in Zeolite 4A Membranes. The Journal of Physical Chemistry B. 2009; 113(13):4267-74. http://dx.doi. org/10.1021/jp805923k

22. Xu X, Wang $\mathbf{J}$ and Long Y. Zeolite-based Materials for Gas Sensors. Sensors. 2006; 6(12):1751-64. http://dx.doi. org/10.3390/s6121751

23. Orlov A, Ozkan S and Karpacheva G. Oxidative polymerization of diphenylamine: A mechanistic study. Polymer Science Series B. 2006; 48(1):11-7. http://dx.doi.org/10.1134/ S1560090406010039

24. Sathiyanarayanan S, Muthukrishnan S and Venkatachari G. Synthesis and anticorrosion properties of polydiphenylamine blended vinyl coatings. Synthetic Metals. 2006; 156(18-20):120812. http://dx.doi.org/10.1016/j.synthmet.2006.08.008

25. Athawale AA, Deore BA and Chabukswar VV. Studies on poly(diphenylamine) synthesized electrochemically in nonaqueous media. Materials Chemistry and Physics. 1999; 58(1):94-100. http://dx.doi.org/10.1016/ S0254-0584(98)00258-2

26. De Santana H and Dias FC. Characterization and properties of polydiphenylamine electrochemically modified by iodide species. Materials Chemistry and Physics. 2003; 82(3):882. http://dx.doi.org/10.1016/j.matchemphys.2003.07.005

27. George E and Christidis HP. Synthesis of FAU Type Zeolite Y from Natural Raw Materials: Hydrothermal SiO2Sinter and Perlite Glass. The Open Mineralogy Journal. 2008; 2(1):1-5. http://dx.doi.org/10.2174/1874456700802010001

28. Kondru AK, Pradeep K and Shri C. Catalytic wet peroxide oxidation of azo dye (Congo red) using modified Y zeolite as catalyst. Journal of Hazardous Materials. 2009; 166(1):342-7. http://dx.doi.org/10.1016/j.jhazmat.2008.11.042

29. Archer M, Christophersen M and Fauchet PM. Electrical porous silicon chemical sensor for detection of organic solvents. Sensors and Actuators B: Chemical. 2005; 106(1):347-57. http://dx.doi.org/10.1016/j.snb.2004.08.016

30. Smallwood IM. Handbook of organic solvent properties. New York: John Wiley \& Sons, Inc.; 1996.

31. Lu L, Xie G, Jiang Y, Du X and Sun P. Organic vapor adsorption behavior of poly (3-henxylthiophene) films on quartz crystal microbalance. Proceeding of International Conference on Optical Instruments and Technology:Advanced Sensor 
Technologies and Applications. 2009; 7508(1). http://dx.doi. org/10.1117/12.837934

32. Xu L, Hu X, Tze Lim Y and Subramanian VS. Organic vapor adsorption behavior of poly(3-butoxythiophene) LB films on quartz crystal microbalance. Thin Solid Films. 2002; 417(1-2):90-4. http://dx.doi.org/10.1016/S00406090(02)00634-X

33. Jose KA, Biju P, Ashwin W, Vijay KV and Reddy CC. A compact wireless gas sensor using a carbon nanotube/ PMMA thin film chemiresistor. Smart Materials and Structures. 2004; 13(5):1045. http://dx.doi.org/10.1088/0964$1726 / 13 / 5 / 010$

34. Auerbach SM, Carrado KA and Dutta PK. Handbook of Zeolite Science and Technology. M. Dekker; 2003. http://dx.doi. org/10.1201/9780203911167

35. Freund MS, Deore B. Self-doped conducting polymers. Wiley; 2007. http://dx.doi.org/10.1002/9780470061725

36. Ayad MM, El-Hefnawey G and Torad NL. Quartz crystal microbalance sensor coated with polyaniline emeraldine base for determination of chlorinated aliphatic hydrocarbons. Sensors and Actuators B: Chemical. 2008; 134(2):887-94. http://dx.doi.org/10.1016/j.snb.2008.06.058

37. Jiang L, Jun H-K, Hoh Y-S, Lim J-O, Lee D-D and Huh J-S. Sensing characteristics of polypyrrole-poly(vinyl alcohol) methanol sensors prepared by in situ vapor state polymerization. Sensors and Actuators B: Chemical. 2005; 105(2):132-7. http:// dx.doi.org/10.1016/j.snb.2003.12.077

38. Tukker A and Simons ILP. Dichloromethane: Advantages and drawbacks of possible market restrictions in the EU. 1999. TNO Report STB - 99-53 Final.

39. Satcher D. Immediately Dangerous To Life or Health (IDLH). Centers for Disease Control and Prevention; 1994 . Available from: <http://www.cdc.gov/niosh/idlh/intridl4.html>.

40. Silverstein RM, Webster FX and Kiemle D. Spectrometric Identification of Organic Compounds. New York: John Willey \& Sons, Inc.; 2005.
41. Lambert JB, Gronert S, Shurvell HF and Lightner D. Organic Structural Spectroscopy. Prentice Hall PTR; 2010.

42. Martin HP, Muller E, Richter R, Roewer G and Brendler E. Conversion process of chlorine containing polysilanes into silicon carbide: Part I Synthesis and crosslinking of poly(chloromethyl)silanes-carbosilanes and their transformation into inorganic amorphous silicon carbide. Journal of Materials Science. 1997; 32(5):1381-7. http://dx.doi. org/10.1023/A:1018577111536

43. Anitha $\mathrm{G}$ and Subramanian E. Recognition and exposition of intermolecular interaction between $\mathrm{CH}_{2} \mathrm{Cl}_{2}$ and $\mathrm{CHCl}_{3}$ by conducting polyaniline materials. Sensors and Actuators B: Chemical. 2005; 107:605-15. http://dx.doi.org/10.1016/j. snb.2004.11.027

44. Vivekanandan JPV, Mahudeswaran A and Vijayanand PS. Synthesis, characterization and conductivity study of polyaniline prepared by chemical oxidative and electrochemical methods. Archives of Applied Science Research. 2011; 3(6):147.

45. De Santana H and Temperini MLA. The Spectroscopic Charactarization of Polydiphenylamine and one of its Oligomeric Fraction. Journal of Brazilian Chemical Society. 1996; 7(6):485-90. http://dx.doi.org/10.5935/01035053.19960087

46. Bennur TH, Srinivas D and Sivasanker S. Oxidation of ethylbenzene over "neat" and zeolite-Y-encapsulated copper tri- and tetraaza macrocyclic complexes. Journal of Molecular Catalysis A: Chemical. 2004; 207(2):163-71. http://dx.doi. org/10.1016/S1381-1169(03)00502-8

47. Choi SY, Park YS, Hong SB and Yoon KB. Iodine as a Visible Probe for the Evaluation of Zeolite Donor Strength. Journal of the American Chemical Society. 1996; 118(39):9377-86. http://dx.doi.org/10.1021/ja952112+

48. Thuwachaowsoan K, Chotpattananont D, Sirivat A, Rujiravanit $\mathrm{R}$ and Schwank JW. Electrical conductivity responses and interactions of poly(3-thiopheneacetic acid)/zeolites L, mordenite, beta and $\mathrm{H}_{2}$. Materials Science and Engineering: B. 2007; 140(1-2):23-30. http://dx.doi.org/10.1016/j. mseb.2007.03.005 